\title{
BMJ Open Effectiveness of a mobile preconception lifestyle programme in couples undergoing in vitro fertilisation (IVF): the protocol for the PreLiFe randomised controlled trial (PreLiFe-RCT)
}

\author{
Tessy Boedt, ${ }^{\oplus, 2}$ Eline Dancet, ${ }^{\circledR 2,3}$ Sharon Lie Fong, ${ }^{2}$ Karen Peeraer, ${ }^{2,3}$ \\ Diane De Neubourg, ${ }^{4}$ Sofie Pelckmans, ${ }^{5}$ Arne van de Vijver, ${ }^{6}$ Jan Seghers, ${ }^{7}$ \\ Katleen Van der Gucht, ${ }^{8,9}$ Ben Van Calster, ${ }^{3,10}$ Carl Spiessens, ${ }^{2,3}$ \\ Christophe Matthys ${ }^{1,11}$
}

To cite: Boedt T, Dancet E, Lie Fong $\mathrm{S}$, et al. Effectiveness of a mobile preconception lifestyle programme in couples undergoing in vitro fertilisation (IVF): the protocol for the PreLiFe randomised controlled trial (PreLiFe-RCT). BMJ Open 2019;9:e029665. doi:10.1136/ bmjopen-2019-029665

- Prepublication history for this paper is available online. To view these files, please visit the journal online (http://dx.doi. org/10.1136/bmjopen-2019029665).

Received 4 February 2019 Accepted 11 June 2019

D) Check for updates

(c) Author(s) (or their employer(s)) 2019. Re-use permitted under CC BY-NC. No commercial re-use. See rights and permissions. Published by BMJ.

For numbered affiliations see end of article.

\section{Correspondence to}

Professor Christophe Matthys; christophe.matthys@uzleuven. be

\section{ABSTRACT}

Introduction Infertility and in vitro fertilisation (IVF; with or without intracytoplasmic sperm injection) result in considerable emotional and financial burden. Increasing evidence suggests that lifestyle factors, including diet, physical activity and personal well-being, are associated with IVF-success rates. Currently, IVF is not routinely combined with a lifestyle programme. The preconception lifestyle (PreLiFe) randomised controlled trial (RCT) assesses the effects of a new mobile PreLiFe programme in couples undergoing IVF.

Methods and analysis A multicentre RCT including 460 heterosexual couples starting IVF in Belgian fertility clinics. IVF couples are randomised between an attention control group or the PreLiFe programme for a period of 12 months or until an ongoing pregnancy is confirmed by ultrasound. The attention control programme includes a mobile application with treatment information (ie, appointments and medication instructions) in addition to standard care. The PreLiFe programme includes a mobile application with the same treatment information in combination with a lifestyle programme. This new lifestyle programme includes tailored advice and skills training on diet, physical activity and mindfulness in combination with text messages and telephone interaction with a healthcare professional trained in motivational interviewing. The primary outcome of this RCT is the cumulative ongoing pregnancy rate within 12 months after randomisation. Secondary outcomes include changes in diet, physical activity, emotional distress, body mass index, waist circumference, quality of life and other reproductive outcomes including IVF discontinuation, clinical pregnancy rate and time to pregnancy. Additionally, partner support and the feasibility (use and acceptability) of the PreLiFe programme will be evaluated in the intervention group. Analysis will be according to intention to treat.

Ethics and dissemination This study has been approved by the Medical Ethical Committee of the Leuven University Hospital (Belgium) and the other recruiting clinics. The findings of this RCT will be disseminated through presentations at international scientific meetings and peerreviewed publications.

\section{Strengths and limitations of this study}

- This is an adequately powered multicentre randomised controlled trial.

- The development of the preconception lifestyle (PreLiFe) programme was theory and evidence based.

- Both partners are included as infertility is a condition affecting couples.

- This is an open-label study, only the statistician is blinded, which can be considered a limitation.

- Due to clinical practice, there is no fixed lead in time free from in vitro fertilisation (IVF), leading for some couples to little time to have an effect of the PreLiFe programme before IVF.

Trial registration number NCT03790449; Pre-results.

\section{INTRODUCTION}

Infertility, defined as failure to achieve clinical pregnancy after 12 months or more of regular unprotected sexual intercourse, affects 1 in 10 heterosexual couples and about half of them seeks fertility treatment. ${ }^{1}$ Infertility and its treatment, including in vitro fertilisation (IVF) with or without intracytoplasmic sperm injection (ICSI), result in considerable emotional and financial burden. ${ }^{2}$ In Belgium, the IVF-success rate, that is, a live born baby is approximately $50 \%$ after 1 year of treatment. ${ }^{45}$ However, during this period, one out of three couples discontinue IVF, mainly due to the IVF-related burden. ${ }^{4}$ Improving IVF-success rates and reducing the burden of IVF are, therefore, important research priorities for reproductive medicine. ${ }^{7}$ 
One potential option for improving IVF-success rates and reducing the burden of IVF is an interdisciplinary developed lifestyle programme. Observational and interventional studies have recently shown that a healthy lifestyle is not only beneficial for infertile patients' general health but also for their IVF-success rate and for reducing IVF burden. More specifically, observational studies showed that couples' healthy diet, normal body mass index (BMI) and moderate physical activity are associated with increased IVF-pregnancy rates. ${ }^{8-15}$ One non-randomised controlled trial (RCT) reports improved diet, physical activity and increased pregnancy rates in infertile women receiving lifestyle education on diet and physical activity in addition to IVF. ${ }^{16}$ Regarding personal well-being, two meta-analyses of observational studies came to contradictory conclusions on whether couples' personal well-being is associated with their IVF outcome. ${ }^{17} 18$ A meta-analysis of interventional studies recently concluded that psychosocial interventions for couples undergoing IVF are effective, both in reducing emotional distress and in improving IVF-pregnancy rates. ${ }^{19}$ Psychosocial interventions focussing on mindfulness are promising as it has recently been shown to result in significant improvements in the fertility-related quality of life of women and in IVF-pregnancy rates. ${ }^{20}$ A guideline of the European Society of Human Reproduction and Embryology highlighted the importance of interdisciplinary support programmes, which can be provided by all staff members during routine fertility care. ${ }^{21}$ So far, no lifestyle programme is offered routinely to IVF couples and this results in one out of three couples deciding for themselves to seek complementary therapy outside of the fertility clinic, including lifestyle and/or psychosocial support. ${ }^{22} 23$

Mobile health (mHealth) as mode of delivery of support programmes has been recognised by (inter)national policy-makers as a promising method for promoting healthy behaviour in both the general population and couples trying to conceive. ${ }^{24-26} \mathrm{~A}$ recent Dutch study showed that a mHealth intervention, targeting among others diet and physical activity of the population of reproductive age, improved their lifestyle and pregnancy rate, especially if both partners participated. ${ }^{14}{ }^{25}$ Nevertheless, no mobile preconception lifestyle programme addressing both infertile men and women and integrating advice on diet and physical activity with mindfulness exercises is available in routine fertility care.

\section{METHODS AND ANALYSIS}

This protocol was based on the Standard Protocol Items: Recommendations for Interventional Trials guidelines. ${ }^{27}$

\section{Aim}

The PreLiFe-RCT aims to assess the effects of a new mobile preconception lifestyle programme for couples undergoing IVF, the PreLiFe programme. This RCT hypothesises that following the PreLiFe programme results in a higher cumulative ongoing pregnancy rate
(COPR) within 12 months as compared with an attention control group.

\section{Study design, setting and timing}

The PreLiFe-RCT is a non-commercial RCT in which the fertility clinics of the following five Belgian hospitals are involved: University Hospitals Leuven, Antwerp University Hospital, Imelda Hospital Bonheiden, General Hospital Diest and General Hospital Sint-Jan Bruges. Eligible couples starting IVF are randomised (1:1 allocation ratio) to the PreLiFe programme or to an attention control group for a period of 12 months or until an ongoing pregnancy is confirmed by ultrasound at 12 weeks of gestational age. Recruitment started in January 2019.

\section{Recruitment}

The treating gynaecologist introduces the study to eligible couples during the consultation prior to starting IVF. Couples who are interested are referred to a researcher, who explains the PreLiFe-RCT in detail and asks the couples for written informed consent. The multicentre set-up of the study ensures that a sufficient number of participants can be included.

\section{Inclusion criteria}

Dutch speaking infertile heterosexual couples starting a first IVF cycle (with or without ICSI; irrespective of the IVF indication), in which the women are maximally 38 years old and in which both partners have a smartphone are eligible.

\section{Exclusion criteria}

Couples, who were previously treated with IVF and/or who need preimplantation genetic testing or donor gametes, are not eligible. In addition, couples are excluded if one of the partners has special dietary requirements due to among others bariatric surgery, coeliac disease or renal disease and/or has movement constraints due to among others cerebral palsy or hemiparesis.

\section{Randomisation, blinding and treatment allocation}

Block randomisation (stratified by clinic) with a 1:1 allocation ratio of eligible, consenting couples is performed with the aid of an online password-protected programme to prevent disclosing the allocation sequence to recruiters. In view of the nature of the intervention, this is an openlabel study where only the statistician is blinded.

\section{Interventions}

During the first 12 months after randomisation or until an ongoing pregnancy is confirmed by ultrasound at 12 weeks of gestational age, participating couples receive standard medical treatment, that is, IVF with or without ICSI according to the local protocol of the participating hospital and without guidance on lifestyle.

Both partners of couples randomised to the control group additionally receive an attention control programme, which mimics the amount of attention received by the intervention group, but is thought not 
to have a specific effect. ${ }^{28}$ More specifically, the attention control group receives a mobile application (app) with treatment information detailing medication instructions and planned appointments.

Both partners of couples randomised to the intervention group additionally receive the new PreLiFe programme. The PreLiFe programme has been developed at KU Leuven, after following multiple steps for developing complex health promotion interventions in line with theory and evidence and after consulting patients and healthcare professionals. ${ }^{29}{ }^{30}$ The main theory followed to improve healthy lifestyle behaviour is the self-determination theory, which requires meeting participants need for autonomy, competence and relatedness. ${ }^{31}$ The PreLiFe programme includes a mobile application (PreLiFe-app) with treatment information and tailored advice and skills training on diet, physical activity and mindfulness in combination with (ie, blended care) interaction with a healthcare professional, trained in motivational interviewing. ${ }^{32}{ }^{33}$ Regarding diet, the PreLiFe-app focusses on improving food literacy, which is described as an interrelated combination of knowledge, skills and self-efficacy on food planning, selecting foods, food preparation, eating and evaluating information about food. ${ }^{345}$ Food literacy is an evidence-based model to develop a lifelong healthy, sustainable and gastronomic relationship with food. The PreLiFe-app tailors the dietary advice and skills with the aid of a limited set of questions on food literacy, resulting in tailored goals, tips and recipes. Regarding physical activity, the PreLiFe-app focusses on improving daily physical activity (at moderate intensity) and reducing sedentary behaviour as advised by WHO. ${ }^{36}$ The physical activity advice and skills training is tailored based on a pedometer linked to the PreLiFe-app and a limited set of questions on the PreLiFe-app, resulting in tailored goals and tips. To improve personal well-being, an evidence-based mindfulness programme is included in the PreLiFe-app. ${ }^{37}{ }^{38}$ The mindfulness exercises follow the format and content of mindfulness-based stress reduction. ${ }^{39-41}$ Participants are instructed to select specific guided exercises based on their own time schedule. The advice and skills training of the different components has different formats including: movies (animation and talking heads), audio files, text supported by graphic figures and photos. Blended care is implemented by allowing couples to ask lifestyle-related questions via text messages in the PreLiFe-app and couples receive a telephone call every 3 months (1, 4, 7 and 10 months after randomisation).

\section{Outcomes, data collection and data management}

The primary outcome of this RCT is COPR within 12 months after randomisation. The secondary biomedical outcomes are: BMI, waist circumference, IVF discontinuation, clinical pregnancy rate and time to pregnancy. The secondary outcomes in which changes are assessed with patient-reported outcome measures are: diet, physical activity, emotional distress and quality of life. In the intervention group, partner support and the feasibility of the PreLiFe programme (ie, use and acceptability) are additionally evaluated. Table 1 describes outcomes, definitions of outcomes, methods of assessment and timings of assessments for each outcome. Data are extracted from medical records, self-administered online questionnaires, the PreLiFe-app or additionally assessed by the researchers (ie, BMI and waist circumference). Local researchers will enter all data in the Good Clinical Practice (GCP) compliant Electronic Data Capture (EDC) platform, 'Castor EDC'. ${ }^{42}$ The combination of this web-based, instantaneous electronic validation and regular on-site monitoring safeguards quality and completeness of the data.

\section{Participant timeline}

Figure 1 provides an overview of all PreLiFe-RCT procedures from recruitment, until the end of the study. Couples, who consented during their consultation prior to IVF, receive a PreLiFe-RCT intake on the same day of their IVF intake. The PreLiFe-RCT intake consists of the following elements: addressing questions of couples about the study; collecting baseline measurements, extracting patients' medical and fertility-related history from medical records; randomisation and configuring the PreLiFe programme. At baseline, 3, 6, 9 and 12 months after randomisation, the researcher sends a link with self-administered online questionnaires on lifestyle behaviour and partner support to participating couples through email and through the mobile app. The follow-up measurements of physical health including height, weight and waist circumference are planned about every 3 months, simultaneously with standard appointments during fertility treatment. Reminders are sent to participants to ensure attendance at follow-up and prevent drop-out of the study. A deviation of 2 weeks before and up to 2 weeks after the planned time of measurement is allowed. IVF trajectories include two different phases. Phase 1, where all couples undergo a fresh IVF cycle and phase 2 with possible pregnancies, follow-up frozen-thawed embryo transfer cycles (if available) and subsequent fresh cycles for which planning differs in time for all couples (see figure 1). The course and outcome of the treatment of the couples is extracted from medical records by the researcher for a period up to 12 months after randomisation. The study ends 12 months after randomisation or if an ongoing pregnancy confirmed by ultrasound (at 12 weeks of gestational age) occurs within 12 months after randomisation. All pregnancies (spontaneous and IVF pregnancies) conceived within these 12 months are followed up until the 12 weeks ultrasound scan. At the end of the study period, the feasibility (use and acceptability) of the PreLiFe programme will be assessed in the intervention group through self-administered online questionnaires. App-based tracking is used throughout the study to evaluate the use of the PreLiFe programme. Participants can withdraw from 
Table 1 Outcomes, definitions of outcomes, methods of assessment and timings of assessments for each outcome

\begin{tabular}{|c|c|c|c|c|c|c|c|}
\hline \multirow[b]{2}{*}{ Outcomes } & \multirow[b]{2}{*}{ Definitions/methods of assessment } & \multicolumn{6}{|c|}{ Timing of assessments } \\
\hline & & Baseline & 3 months & 6 months & 9 months & 12 months & Continuously \\
\hline $\begin{array}{l}\text { Patient-reported } \\
\text { outcome measures }\end{array}$ & $\begin{array}{l}\text { Questionnaire name (abbreviation) } \\
\text { Content of questions } \\
\text { Details on evaluation, subscales and scoring }\end{array}$ & & & & & & \\
\hline $\begin{array}{l}\text { Background and } \\
\text { general lifestyle } \\
\text { behaviour }\end{array}$ & $\begin{array}{l}\text { Questions on background and general lifestyle behaviour. } \\
\text { Questions on smoking, alcohol use, supplement intake } \\
\text { and complementary therapy. } \\
\text { Descriptive evaluation. }\end{array}$ & $x$ & $x$ & $x$ & $x$ & $x$ & \\
\hline Diet & $\begin{array}{l}\text { Food Frequency Questionnaire (FFQ). }{ }^{49} \\
\text { Questions on frequency and portion size of consumption } \\
\text { of foods and beverages. } \\
\text { Evaluation of dietary pattern and diet quality (index to } \\
\text { reflect compliance with food based dietary guidelines }{ }^{50} \text { ). } \\
\text { Diet quality score: } 0-100 \text { (the higher, the better diet } \\
\text { quality). }\end{array}$ & $x$ & $x$ & $x$ & $x$ & $x$ & \\
\hline Physical activity & $\begin{array}{l}\text { International Physical Activity Questionnaire Short Form (IPAQ- } \\
\text { SF). }{ }^{51} \\
\text { Questions on duration and frequency of different } \\
\text { intensities of physical activity. } \\
\text { Evaluation based on WHO recommendations. }{ }^{36}\end{array}$ & $x$ & $x$ & $x$ & $x$ & $x$ & \\
\hline Personal well-being & $\begin{array}{l}\text { Depression, Anxiety and Stress Scale (DASS-21). }{ }^{52} 53 \\
\text { Questions on symptoms of stress, anxiety and depression } \\
\text { (emotional distress). } \\
\text { Stress, anxiety and depression subscales, overall score: } \\
0-126 \text { (the higher, the more emotional distress). }\end{array}$ & $x$ & $x$ & $x$ & $x$ & $x$ & \\
\hline Quality of life (QOL) & $\begin{array}{l}\text { Fertility Quality of Life Tool (FERTIQOL) } .^{5455} \\
\text { Questions on fertility-related QOL. } \\
\text { Emotional, mind-body, relational and social subscales, } \\
\text { overall score: } 0-100 \text { (the higher, the better QOL). }\end{array}$ & $x$ & $x$ & $x$ & $x$ & $x$ & \\
\hline
\end{tabular}

Partner support * $\quad \begin{aligned} & \text { Questionnaire based on the social support for diet and } \\ & \text { exercise scales. }\end{aligned}$
Questions on partner support for diet, physical activity and
mindfulness.
Support for diet $(0-15)$, physical activity $(0-15)$ and
mindfulness $(0-10)$ subscales (the higher, the better
partner support).

Acceptability of A short version of the subjective quality subscale of the Mobile

PreLiFe programme App Rating Scale (MARS). ${ }^{57}$

- Questions on the acceptability and subjective quality of the PreLiFe programme.

- Descriptive evaluation+subjective quality: 0-10 (the higher the better subjective quality of the PreLiFe programme).

\begin{tabular}{|c|c|c|c|c|c|c|c|}
\hline $\begin{array}{l}\text { Outcomes collected } \\
\text { from PreLiFe-app }\end{array}$ & Definition/specification & & & & & & \\
\hline $\begin{array}{l}\text { Use of } \\
\text { PreLiFe programme* }\end{array}$ & $\begin{array}{l}\text { App-based tracking to evaluate the percentage of participants } \\
\text { (couples) using the PreLiFe programme in combination } \\
\text { with a question on their motivation of (not) using the } \\
\text { PreLiFe programme. }\end{array}$ & & $x$ & $x$ & $x$ & $x$ & $\mathrm{x}$ \\
\hline $\begin{array}{l}\text { Outcomes extracted } \\
\text { from medical records }\end{array}$ & Definition/specification & & & & & & \\
\hline $\begin{array}{l}\text { Sociodemographic } \\
\text { background }\end{array}$ & Age; ethnicity; level of education; profession. & $x$ & & & & & \\
\hline Medical history & $\begin{array}{l}\text { Current and resolved medical conditions; current medication } \\
\text { use. }\end{array}$ & $\mathrm{x}$ & & & & & \\
\hline Fertility history & $\begin{array}{l}\text { Duration of self-reported infertility; indication of infertility: } \\
\text { male, female or mixed factor infertility; primary or secondary } \\
\text { infertility. }\end{array}$ & $x$ & & & & & \\
\hline $\begin{array}{l}\text { Course of IVF } \\
\text { treatment }\end{array}$ & $\begin{array}{l}\text { Details on fresh and frozen-thawed IVF/ICSI cycles such as } \\
\text { date and type of stimulation, date of aspiration, no of oocytes, } \\
\text { total motile sperm count, date of fresh embryo transfer, date } \\
\text { of frozen-thawed embryo transfer, in case of a cancelled } \\
\text { cycle, date and reason of cancellation; outcome of the cycle } \\
\text { (detection of hCG) and any adverse events. }\end{array}$ & & & & & & $\mathrm{x}$ \\
\hline Clinical pregnancy & $\begin{array}{l}\text { A pregnancy diagnosed by ultrasonographic visualisation } \\
\text { of one or more gestational sacs or definitive clinical signs of } \\
\text { pregnancy. }\end{array}$ & & & & & & $\mathrm{x}$ \\
\hline $\begin{array}{l}\text { Time to (clinical) } \\
\text { pregnancy }\end{array}$ & $\begin{array}{l}\text { The time taken to establish a pregnancy, measured in } \\
\text { months. }{ }^{58}\end{array}$ & & & & & & $\mathrm{x}$ \\
\hline
\end{tabular}


Table 1 Continued

\begin{tabular}{|c|c|c|c|c|c|c|c|}
\hline \multirow[b]{2}{*}{ Outcomes } & \multirow[b]{2}{*}{ Definitions/methods of assessment } & \multicolumn{6}{|c|}{ Timing of assessments } \\
\hline & & Baseline & 3 months & 6 months & 9 months & 12 months & Continuously \\
\hline Ongoing pregnancy & $\begin{array}{l}\text { A viable intrauterine pregnancy of at least } 12 \text { weeks duration } \\
\text { confirmed on ultrasound scan. }{ }^{59}\end{array}$ & & & & & & $\mathrm{x}$ \\
\hline IVF discontinuation & $\begin{array}{l}\text { Couples who had quit IVF before the achievement of a } \\
\text { pregnancy. }\end{array}$ & & & & & & $\mathrm{x}$ \\
\hline $\begin{array}{l}\text { Outcomes measured } \\
\text { by the researcher }\end{array}$ & Definition/specification & & & & & & \\
\hline $\begin{array}{l}\text { Body mass } \\
\text { index (BMI) }\end{array}$ & $\begin{array}{l}\text { To estimate nutritional status. BMI is defined as a person's } \\
\text { weight in kilograms divided by the square of the person's } \\
\text { height in metres }\left(\mathrm{kg} / \mathrm{m}^{2}\right) \text {. } \\
\text { Weight is measured when wearing light clothes and no shoes } \\
\text { on a calibrated scale } \\
\text { Height is measured without shoes on a stadiometer. }\end{array}$ & $x$ & $x$ & $x$ & $x$ & $x$ & \\
\hline Waist circumference & $\begin{array}{l}\text { To estimate abdominal fat. } \\
\text { Waist circumference is measured with a waist circumference } \\
\text { measuring tape according to international Standards for } \\
\text { Anthropometric Assessment. }\end{array}$ & $x$ & $x$ & $x$ & $x$ & $x$ & \\
\hline
\end{tabular}

*Only measured in the intervention group.

hCG, human chorionic gonadtropin; ICSI, intracytoplasmic sperm injection; IVF, in vitro fertilisation.

the study at any time for any reason if they wish to do so without any consequences on their IVF trajectory.

\section{Sample size}

A sample size for an intention-to-treat analysis of the primary outcome (COPR) was calculated, in collaboration with a statistician from KU Leuven. The calculations were based on literature from the field of reproductive medicine regarding: (1) the optimistic, realistic and pessimistic cumulative IVF-success rates in Belgium, ${ }^{45}$ (2) the IVF-discontinuation rates in Belgium, ${ }^{4}$ (3) data on the impact of a preconception lifestyle intervention on IVF-success rates $^{16}(4)$ data on the impact of a psychosocial intervention on IVF-discontinuation rates ${ }^{43}$ and (5) data on withdrawal of fertility patients from lifestyle interventions. ${ }^{43} 44$ Assuming a COPR of $50 \%$ in the control group ${ }^{45}$ and $63 \%$ in the intervention group dictates a sample size of 230 couples per group or 460 couples in total (two-sided test; power of $80 \%$ and alpha of $5 \%$ ). The $13 \%$ increase in COPR within the first 12 months after starting IVF is partly expected by assuming improved IVF-success rates and partly by assuming decreased IVF-discontinuation rates. More specifically, a lifestyle programme targeting physical

Participants

Groups (Control \& Intervention)

Outcomes

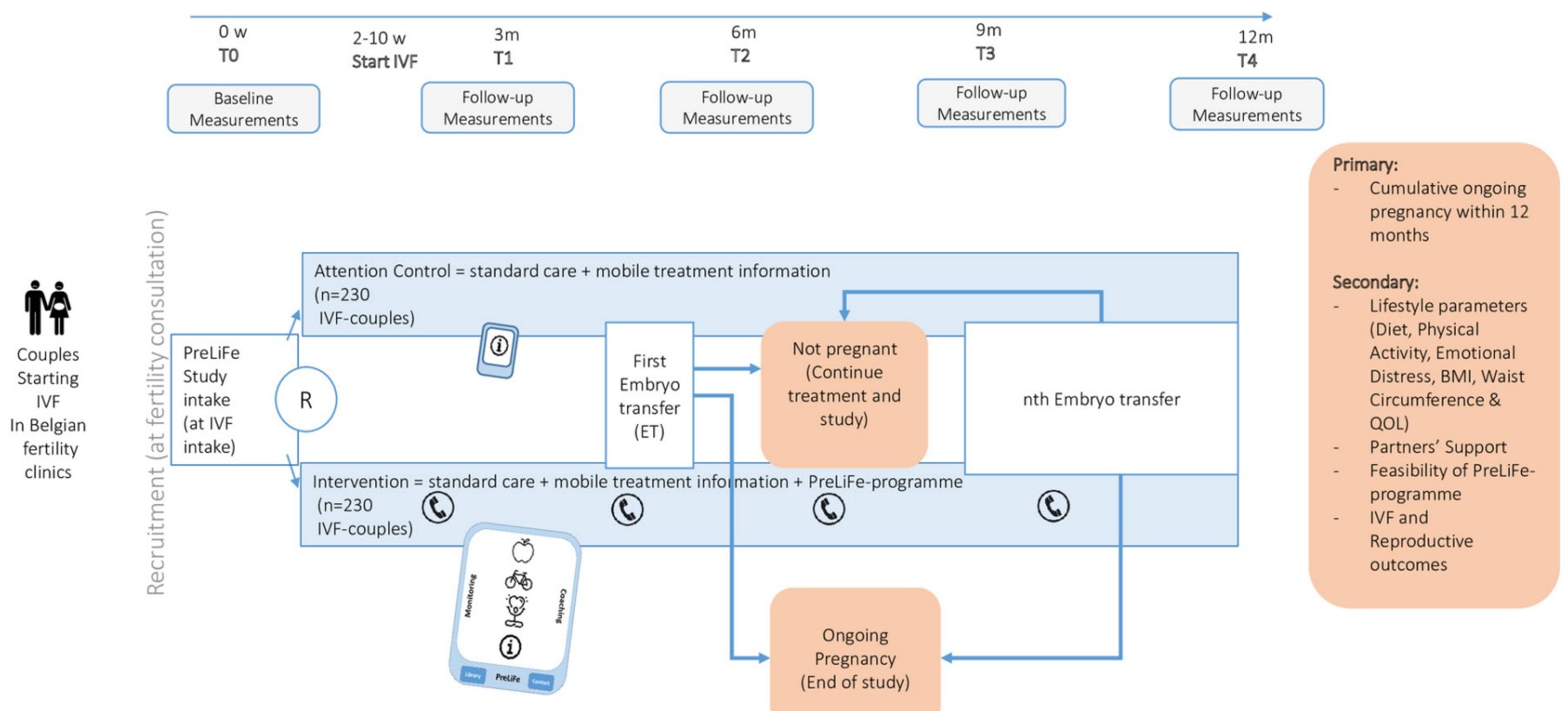

Figure 1 Overview of PreLiFe-RCT. BMI, body mass index; IVF, in vitro fertilisation; QOL, quality of life; RCT, randomised controlled trial. 
activity, diet and stress management increased the clinical pregnancy rates of one IVF cycle from $19.2 \%$ to $46.1 \%{ }^{16}$ Regarding decreasing IVF-discontinuation rates, a cognitive coping and relaxation programme had a tendency to decrease the IVF-discontinuation rate within 12 months from $15.2 \%$ to $5.5 \%{ }^{43}$ Calculations were performed using PASS V.14 software. ${ }^{45}$

\section{Data analysis}

Analysis will be according to the intention to treat. Descriptive statistics for baseline characteristics in the two arms will be presented and the withdrawal rate from the study will be assessed and compared between the two arms. The primary outcome is COPR within 12 months after randomisation. To calculate this, an ongoing pregnancy conceived within 12 months after randomisation will be counted as a positive event, whereas IVF discontinuation and absence of pregnancy will be counted as a negative event. The COPR in both groups will be compared using multivariate logistic regression models with controlling for potential confounders such as age and BMI. ORs with $95 \%$ CIs will be reported. A $p<0.05$ will be used to determine statistical significance for the intervention. Furthermore, cumulative incidences of ongoing pregnancy and IVF discontinuation in the intervention and control group will be described. Similar analysis will be performed for binary secondary outcomes such as clinical pregnancy. Additionally, we will evaluate changes in lifestyle parameters including changes in the diet, physical activity, emotional distress, BMI, waist circumference and fertility-related quality of life over time and we will evaluate the differences between the intervention and control group in these parameters. Mixed models for repeated measurements (MMRM) will be used to evaluate treatment, time and interactive effects on these secondary outcomes. The determination of statistical significance will not be central to the analysis of secondary endpoints, yet nominal $p$ values may be reported. Descriptive analysis will be conducted on additional parameters measured only in the intervention group, more specifically: partner support and feasibility of the PreLiFe programme. Regarding missing data, MMRM is used which is consistent under the 'missing at random' assumption and in line with the intention-to-treat principle. ${ }^{46}$ For the primary outcome, we do not expect missing data.

\section{Harms}

Throughout the PreLiFe-RCT, all solicited and spontaneously reported adverse events and other unintended effects of the PreLiFe programme or RCT will be collected, assessed, reported and managed according to GCP.

\section{Patient and public involvement}

For the development of the PreLiFe programme and the PreLiFe-RCT, we applied a human-centred design, consulting both patients and healthcare professionals. Additionally, an advisory committee has been installed from the start of the development of the project and includes representatives of the Belgian patient association 'De Verdwaalde Ooievaar' and of the 'Belgian Society for Reproductive Medicine'.

\section{DISSEMINATION}

Confidentiality of the participant's data is ensured by using participant IDs rather than identifiable information in the data set (ie, coding) and by storing the document linking the IDs to the identifiable information separately. Only researchers from the study have access to the coded data.

The findings of this RCT will be disseminated through presentations at international scientific meetings and in peer-reviewed publications in accordance with academic standards. The participating sites are not allowed to publish any data or results from the study prior the multicentre publication. Authorship to publications will be in accordance with the requirements published by the International Committee of Medical Journal Editors, in accordance with the requirements of the respective medical journal and according to the KU Leuven Publication Policy. We do not intend to collaborate with a medical writer.

\section{DISCUSSION}

The PreLiFe-RCT examines a novel preconception lifestyle programme for couples undergoing IVF, including tailored advice and skills training on diet, physical activity and mindfulness, in a mHealth format combined with motivational interviewing via text messages and telephone interaction. This PreLiFe programme is theory and evidence based and has been developed systematically. ${ }^{30} 47$ Besides examining a novel lifestyle intervention for couples undergoing IVF, with the potential of low-cost widespread implementation, this RCT has several strengths. First, this RCT has adequate power, which is enabled by the multicentre setting. Second, this RCT includes couples rather than individuals in the light of the evidence that addressing couples in lifestyle interventions provides extra support and maximises compliance. ${ }^{25} 48$ Third, this RCT has an attention control condition rather than standard care. ${ }^{28}$ This RCT has also some potential limitations. A limitation, which is inevitable due to the nature of the intervention, is that this is an open-label study where only the statistician could be blinded. A second potential limitation is that due to clinical practice, the PreLiFe programme is offered right before the start of IVF without a fixed lead in time free from IVF. This leads for some couples to little time to follow the PreLiFe programme and improve their lifestyle before their first IVF cycle. However, we will capture the time between offering the PreLiFe programme and start of IVF. Finally, publishing this protocol outlines our effort to limit the risk of bias in our RCT.

With this RCT, we expect to demonstrate the added value of a mobile preconception lifestyle programme for 
reproductive and lifestyle outcomes in couples undergoing IVF. If this RCT proves that our lifestyle programme is effective, lifestyle support programmes should be implemented in standard care in each fertility clinic.

\section{Author affiliations}

${ }^{1}$ Department of Chronic Diseases Metabolism and Ageing, KU Leuven, Leuven, Belgium

${ }^{2}$ Leuven University Fertility Centre, University Hospitals Leuven, Leuven, Belgium ${ }^{3}$ Department of Development and Regeneration, KU Leuven, Leuven, Belgium ${ }^{4}$ Antwerp University Hospital, Centre for Reproductive Medicine, Edegem, Belgium ${ }^{5}$ Department of Obstetrics and Gynaecology, Imelda Hospital Bonheiden, Bonheiden, Belgium

${ }^{6}$ Department of Obstetrics and Gynaecology, General Hospital Sint Jan BrugesOstend, Brugge, Belgium

${ }^{7}$ Department of Movement Sciences, KU Leuven, Leuven, Belgium

${ }^{8}$ Leuven Mindfulness Centre, KU Leuven, Leuven, Belgium

${ }^{9}$ Faculty of Psychology and Educational Sciences, KU Leuven, Leuven, Belgium

${ }^{10}$ Department of Biomedical Data Sciences, Leiden University Medical Centre, Leiden, Netherlands

${ }^{11}$ Department of Endocrinology, University Hospitals Leuven, Leuven, Belgium

Acknowledgements We acknowledge Barbara Weyn and Steve De Backer for the technical development of the PreLiFe-programme; Roos Voorend and Jan Derboven for contributing to the human centred design of the PreLiFe-programme, as well as all the patients, midwives and gynaecologists participating in the human centred design research; Edel Maex, Filip Raes and Peter Kuppens for contributing to the development of the mindfulness part of our PreLiFe-programme; An Bogaerts for contributing to the development of the physical activity part of our PreLiFeprogramme and the Nutrition Unit of University Hospitals Leuven for contributing to the development of the diet part of our PreLiFe-programme. Additionally, we want to acknowledge our advisory committee of our FWO-TBM project and all clinics who are contributing to patient recruitment or are preparing to do so.

Contributors TB, ED, KVdG, JS, BVC, CS and CM designed the trial, developed the protocol and applied for funding. TB, KP, DDN, SP, AvdV and SLF applied for ethical approval and implemented the logistics of the trial. All authors read, revised and approved the final manuscript.

Funding This work was supported by the Research foundation Flanders (Belgium). (FW0-TBM; reference: T005417N).

\section{Competing interests None declared.}

Patient consent for publication Not required.

Ethics approval This study has been approved by the Medical Ethical Committee of the University Hospitals Leuven (Belgium) and the local ethics committees of the participating clinics (ie, Antwerp University Hospital, Imelda Hospital Bonheiden, General Hospital Diest and General Hospital Sint Jan Bruges)(s61596). If any protocol amendments would have to be made, they will be reported and submitted to all medical ethical committees.

Provenance and peer review Not commissioned; externally peer reviewed.

Open access This is an open access article distributed in accordance with the Creative Commons Attribution Non Commercial (CC BY-NC 4.0) license, which permits others to distribute, remix, adapt, build upon this work non-commercially, and license their derivative works on different terms, provided the original work is properly cited, appropriate credit is given, any changes made indicated, and the use is non-commercial. See: http://creativecommons.org/licenses/by-nc/4.0/.

\section{REFERENCES}

1. Boivin J, Bunting L, Collins JA, et al. International estimates of infertility prevalence and treatment-seeking: potential need and demand for infertility medical care. Hum Reprod 2007;22:1506-12.

2. Wu AK, Elliott P, Katz PP, et al. Time costs of fertility care: the hidden hardship of building a family. Fertil Steril 2013;99:2025-30.

3. Gameiro S, Boivin J, Peronace L, et al. Why do patients discontinue fertility treatment? A systematic review of reasons and predictors of discontinuation in fertility treatment. Hum Reprod Update 2012;18:652-69.
4. De Neubourg D, Bogaerts K, Blockeel C, et al. How do cumulative live birth rates and cumulative multiple live birth rates over complete courses of assisted reproductive technology treatment per woman compare among registries? Hum Reprod 2016;31:93-9.

5. Peeraer K, Debrock S, Laenen A, et al. The impact of legally restricted embryo transfer and reimbursement policy on cumulative delivery rate after treatment with assisted reproduction technology. Hum Reprod 2014;29:267-75.

6. Brandes M, van der Steen JO, Bokdam SB, et al. When and why do subfertile couples discontinue their fertility care? A longitudinal cohort study in a secondary care subfertility population. Hum Reprod 2009;24:3127-35.

7. Gameiro S, Boivin J, Domar A. Optimal in vitro fertilization in 2020 should reduce treatment burden and enhance care delivery for patients and staff. Fertil Steril 2013;100:302-9.

8. Piché ML, Babineau V, Robitaille J, et al. Lifestyle-related factors associated with reproductive health in couples seeking fertility treatments: results of a pilot study. Int J Fertil Steril 2018;12:19-26.

9. Vujkovic M, de Vries JH, Lindemans J, et al. The preconception Mediterranean dietary pattern in couples undergoing in vitro fertilization/intracytoplasmic sperm injection treatment increases the chance of pregnancy. Fertil Steril 2010;94:2096-101.

10. Twigt JM, Bolhuis ME, Steegers EA, et al. The preconception diet is associated with the chance of ongoing pregnancy in women undergoing IVF/ICSI treatment. Hum Reprod 2012;27:2526-31.

11. Salas-Huetos A, Bulló M, Salas-Salvadó J, et al. foods and nutrients in male fertility parameters and fecundability: a systematic review of observational studies. Hum Reprod Update 2017:1-19 (published Online First: 2017/03/10).

12. Sundaram R, Mumford SL, Buck Louis GM. Couples' body composition and time-to-pregnancy. Hum Reprod 2017;32:662-8.

13. Rittenberg V, Seshadri S, Sunkara SK, et al. Effect of body mass index on IVF treatment outcome: an updated systematic review and meta-analysis. Reprod Biomed Online 2011;23:421-39.

14. van Dijk MR, Koster MPH, Willemsen SP, et al. Healthy preconception nutrition and lifestyle using personalized mobile health coaching is associated with enhanced pregnancy chance. Reprod Biomed Online 2017;35:453-60.

15. Rao M, Zeng Z, Tang L. Maternal physical activity before IVF/ ICSI cycles improves clinical pregnancy rate and live birth rate: a systematic review and meta-analysis. Reprod Biol Endocrinol 2018;16:11

16. Kaya Y, Kizilkaya Beji N, Aydin Y, et al. The effect of health-promoting lifestyle education on the treatment of unexplained female infertility. Eur J Obstet Gynecol Reprod Biol 2016;207:109-14.

17. Boivin J, Griffiths E, Venetis CA. Emotional distress in infertile women and failure of assisted reproductive technologies: meta-analysis of prospective psychosocial studies. BMJ 2011;342:d223.

18. Matthiesen SM, Frederiksen Y, Ingerslev HJ, et al. Stress, distress and outcome of assisted reproductive technology (ART): a metaanalysis. Hum Reprod 2011;26:2763-76

19. Frederiksen Y, Farver-Vestergaard I, Skovgård NG, et al. Efficacy of psychosocial interventions for psychological and pregnancy outcomes in infertile women and men: a systematic review and meta-analysis. BMJ Open 2015;5:e006592.

20. Li J, Long L, Liu Y, et al. Effects of a mindfulness-based intervention on fertility quality of life and pregnancy rates among women subjected to first in vitro fertilization treatment. Behav Res Ther 2016;77:96-104

21. Gameiro S, Boivin J, Dancet E, et al. ESHRE guideline: routine psychosocial care in infertility and medically assisted reproduction-a guide for fertility staff. Hum Reprod 2015;30:2476-85.

22. Porat-Katz A, Paltiel O, Kahane A, et al. The effect of using complementary medicine on the infertility-specific quality of life of women undergoing in vitro fertilization. Int J Gynaecol Obstet 2016;135:163-7.

23. Smith JF, Eisenberg ML, Millstein SG, et al. The use of complementary and alternative fertility treatment in couples seeking fertility care: data from a prospective cohort in the United States. Fertil Steril 2010;93:2169-74.

24. Afshin A, Babalola D, Mclean M, et al. Information Technology and Lifestyle: A Systematic Evaluation of Internet and Mobile Interventions for Improving Diet, Physical Activity, Obesity, Tobacco, and Alcohol Use. J Am Heart Assoc 2016;5.

25. Van Dijk MR, Huijgen NA, Willemsen SP, et al. Impact of an mHealth Platform for Pregnancy on Nutrition and Lifestyle of the Reproductive Population: A Survey. JMIR Mhealth Uhealth 2016;4:e53.

26. van Dongen AJ, Nelen WL, IntHout J, et al. e-Therapy to reduce emotional distress in women undergoing assisted reproductive technology (ART): a feasibility randomized controlled trial. Hum Reprod 2016;31:1046-57. 
27. Chan AW, Tetzlaff JM, Altman DG, et al. SPIRIT 2013 statement: defining standard protocol items for clinical trials. Ann Intern Med 2013;158:200-7.

28. Aycock DM, Hayat MJ, Helvig A, et al. Essential considerations in developing attention control groups in behavioral research. Res Nurs Health 2018;41:320-8.

29. Craig P, Dieppe P, Macintyre S, et al. Developing and evaluating complex interventions: the new Medical Research Council guidance. BMJ 2008;337:a1655.

30. Eldredge LKB MC, Ruiter RAC, Fernandez ME, et al. Planning health promotion programs. An intervention mapping approach. Fourth edition. San Fransisco CA: Jossey-Bass A Wiley Brand, 2016

31. Ryan RM, Deci EL. Self-determination theory and the facilitation of intrinsic motivation, social development, and well-being. Am Psychol 2000:55:68-78

32. Lussier MT, Richard C. The motivational interview: in practice. Can Fam Physician 2007;53:2117-8.

33. Homan G, Litt J, Norman RJ. The FAST study: Fertility ASsessment and advice Targeting lifestyle choices and behaviours: a pilot study. Hum Reprod 2012;27:2396-404.

34. Vidgen HA, Gallegos D. Defining food literacy and its components. Appetite 2014;76:50-9.

35. Azevedo Perry E, Thomas H, Samra HR, et al. Identifying attributes of food literacy: a scoping review. Public Health Nutr 2017;20:2406-15.

36. WHO. Global recommendations on physical activity for health, 2010.

37. Khoury B, Lecomte T, Fortin G, et al. Mindfulness-based therapy: a comprehensive meta-analysis. Clin Psychol Rev 2013;33:763-71.

38. Goyal M, Singh S, Sibinga EM, et al. Meditation programs for psychological stress and well-being: a systematic review and metaanalysis. JAMA Intern Med 2014;174:357-68.

39. Maex E. In de maalstroom van je leven. Belgium: Lannoo Meulenhoff, 2010.

40. Kabat-Zinn J. Full catastrophe living, revised edition: how to cope with stress, pain and illness using mindfulness meditation. Hachette UK, 2013.

41. Crane RS, Brewer J, Feldman C, et al. What defines mindfulnessbased programs? The warp and the weft. Psychol Med 2017;47:990-9.

42. Ciwit BV. Castor Electronic Data Capture. Amsterdam, The Netherlands, 2018. www.castoredc.com.

43. Domar AD, Gross J, Rooney K, et al. Exploratory randomized trial on the effect of a brief psychological intervention on emotions, quality of life, discontinuation, and pregnancy rates in in vitro fertilization patients. Fertil Steril 2015;104:440-51.

44. Mutsaerts MA, van Oers AM, Groen $\mathrm{H}$, et al. Randomized tria of a lifestyle program in obese infertile women. $N$ Engl $J$ Med 2016;374:1942-53.
45. NCSS, LLC. Power Analysis and Sample Size Software version 14.0.7. Kaysville (Utah), USA. ncss.com/software/pass.

46. Molenberghs $\mathrm{G}$, Thijs $\mathrm{H}$, Jansen I, et al. Analyzing incomplete longitudinal clinical trial data. Biostatistics 2004;5:445-64.

47. Craig P, Dieppe P, Macintyre S, et al. Developing and evaluating complex interventions: the new Medical Research Council guidance. Int J Nurs Stud 2013:50:587-92.

48. Best D, Avenell A, Bhattacharya S, et al. New debate: is it time for infertility weight-loss programmes to be couple-based? Hum Reprod 2017;32:2359-65.

49. Matthys C, Meulemans A, Schueren BVD. Development and validation of general FFQ for use in clinical practice. Annals of Nutrition and Metabolism 2015;67(S1):239.

50. Huybrechts I, Vereecken C, De Bacquer D, et al. Reproducibility and validity of a diet quality index for children assessed using a FFQ. Br J Nutr 2010;104:135-44

51. Craig CL, Marshall AL, Sjöström M, et al. International physical activity questionnaire: 12-country reliability and validity. Med Sci Sports Exerc 2003;35:1381-95.

52. de Beurs E, van Dyck R M. De DASS: Een vragenlijst voor het meten van depressie: angst en stress, 2001.

53. Lovibond PF, Lovibond SH. The structure of negative emotional states: comparison of the Depression Anxiety Stress Scales (DASS) with the Beck Depression and Anxiety Inventories. Behav Res Ther 1995;33:335-43.

54. Boivin J, Takefman J, Braverman A. The fertility quality of life (FertiQoL) tool: development and general psychometric properties. Hum Reprod 2011;26:2084-91.

55. Aarts JW, van Empel IW, Boivin J, et al. Relationship between quality of life and distress in infertility: a validation study of the Dutch FertiQoL. Hum Reprod 2011;26:1112-8.

56. Carlson JA, Sallis JF, Wagner N, et al. Brief physical activity-related psychosocial measures: reliability and construct validity. J Phys Act Health 2012;9:1178-86.

57. Stoyanov SR, Hides L, Kavanagh DJ, et al. Mobile app rating scale: a new tool for assessing the quality of health mobile apps. JMIR Mhealth Uhealth 2015;3:e27.

58. Zegers-Hochschild F, Adamson GD, Dyer S, et al. The International Glossary on Infertility and Fertility Care, 2017. Hum Reprod 2017;32:1786-801.

59. Braakhekke M, Kamphuis El, Dancet EA, et al. Ongoing pregnancy qualifies best as the primary outcome measure of choice in trials in reproductive medicine: an opinion paper. Fertil Steril 2014;101:1203-4.

60. Verkuijlen J, Verhaak C, Nelen WL, et al. Psychological and educational interventions for subfertile men and women. Cochrane Database Syst Rev 2016;3:Cd011034. 